\title{
FINITELY GENERATED KLEINIAN GROUPS An Introduction
}

\author{
Lipman Bers
}

This paper, like the talk on which it is based, is directed not at workers in the theory of Kleinian groups but at mathematicians who want to find out what this theory is all about. An introductory talk cannot, of course, cover all aspects of a rapidly developing field. We stress those chapters of the theory in which the connections with Riemann surfaces and quasiconformal maps predominate; these are also the chapters in which the influence of Ahlfors is clearly visible. The reader will have to look elsewhere for an introductory account on Thurston's pathbreaking work on hyperbolic three-manifolds. Neither can we report on Maskit's monumental classification of geometrically finite function groups.

For more information, and for references to original papers, I refer to the books and expository papers listed in the selected bibliography.

\section{Basic definitions}

Every unimodular $2 \times 2$ matrix with complex entries,

$$
\alpha=\left(\begin{array}{ll}
a & b \\
c & d
\end{array}\right), \quad a d-b c=1
$$

defines a biholomorphic automorphism

$$
z \mapsto \frac{a z+b}{c z+d}
$$

of the Riemann sphere (= complex projective line) $\hat{\mathbf{C}}=\mathbf{C} \cup\{\infty\}$; the same map is defined by the matrix $(-\alpha)$. The Möbius group $\mathbf{M}$ of all such maps can be identified with the group $\operatorname{PSL}(2, \mathbf{C})$.

Every Möbius transformation (1.1) can be extended to act on the hyperbolic three-space $\mathbf{H}_{3}$. Indeed, $\mathbf{H}_{3}$ can be represented as the space of all "special" quaternions $Z=x+i y+j t$, with $x, y, t$ real and $t>0$. The Möbius transformation (1.1) takes $Z$ into the special quaternion

$$
(a Z+b)(c Z+d)^{-1}
$$


it preserves orientation and the Poincaré metric

$$
d s^{2}=\left(d x^{2}+d y^{2}+d t^{2}\right) / t^{2} .
$$

Similarly, the real Möbius group $\mathbf{M}_{\mathbf{R}}$, which can be identified with $\operatorname{PSL}(2, \mathbf{R})$, is the group of all projective self-mappings of the real projective line $\hat{\mathbf{R}}=\mathbf{R} \cup\{\infty\}$, and also the group of motions of the non-Euclidean plane $\mathbf{H}_{2}$, represented as the upper half-plane $U$ in $\mathbf{C}$, with the Poincaré metric $d s^{2}=\left(d x^{2}+d y^{2}\right) / y^{2}$.

Now let $G$ be a discrete subgroup of $\mathbf{M}$. A point $z_{0} \in \hat{\mathbf{C}}$ is called a point of discontinuity (of $G$ ) if there is a neighborhood of $z_{0}$ in which the elements of $G$ form a normal family. The set of all points of discontinuity is the region of discontinuity $\Omega(G)$ of $G$; its complement $\Lambda(G)=\hat{\mathbf{C}} \backslash \Omega(G)$ is called the limit set. The set of discontinuity is always open and $G$-invariant.

In every $G$-invariant open set $\omega \subset \Omega(G)$ the group $G$ operates properly discontinuously; this means that every compact subset of $\omega$ intersects at most finitely many of its translates under $G$. Since every element of $G$ is a conformal map, one concludes that, for every component $\Delta$ of $\Omega(G)$, the quotient

$$
S_{\Delta}=\Delta / \operatorname{Stab}_{G} \Delta
$$

is a Riemann surface and the canonical surjection $\Delta \rightarrow S_{\Delta}$ is a holomorphic covering, which is branched precisely at those points of $\Delta$ which are fixed under a non-trivial element of $\operatorname{Stab}_{G} \Delta$, the stabilizer of $\Delta$ in $G$. The quotient $\Omega(G) / G$ is the disjoint union

$$
S_{\Delta_{1}}+S_{\Delta_{2}}+\cdots
$$

where $\Delta_{1}, \Delta_{2}, \ldots$ is a complete list of nonconjugate (under $G$ ) components of $\Delta$. We say that $G$ represents $S_{\Delta_{i}}$ over $\Delta_{i}$ and $S_{\Delta_{1}}+S_{\Delta_{2}}+\cdots$ over $\Omega(G)$.

The group $G$ is called Kleinian (by some authors, Kleinian of the second kind) if its limit set is not the whole Riemann sphere $\hat{\mathbf{C}}$. If so, the limit set has either no more than two points (in which case $G$ is called elementary) or infinitely many points (in which case the limit set $\Lambda(G)$ is nowhere dense and perfect).

By a Fuchsian group we shall mean, in this paper, a Kleinian group $G$ which lies in $\mathbf{M}_{\mathbf{R}}$. Such a $G$ is called of the first kind (or of the second kind) if $\Lambda(G)=$ $\hat{\mathbf{R}}=\mathbf{R} \cup\{\infty\}$ (or if $\mathbf{R}$ is a nowhere dense subset of $\hat{\mathbf{R}}$ ). A Fuchsian group $G$ of the first kind represents 2 Riemann surfaces between which there exists an anti-conformal map, induced by the reflection $z \mapsto \bar{z}$. A Fuchsian group of the second kind represents one Riemann surface endowed by a canonical anticonformal involution, induced by the reflection $z \mapsto \bar{z}$. Any Kleinian group $G$ acts properly discontinuously on the non-Euclidean space $\mathbf{H}_{3}$; this is so even if $\Omega(G)=\emptyset$. If $G$ is torsion free, the quotient $\mathbf{H}_{3} / G$ is a manifold endowed with a smooth hyperbolic metric, induced by the Poincare metric in $\mathbf{H}_{3}$. If $G$ has torsion, the natural map $\mathbf{H}_{3} \rightarrow \mathbf{H}_{3} / G$ has branch points. 
The assumption that $G$ has no torsion is often made for the sake of simplicity. This restriction is not too severe, in view of Selberg's lemma: a finitely generated matrix group is either torsion free or has a torsion free subgroup of finite index.

\section{Examples}

A discrete subgroup of $\mathbf{M}_{3}$ for which every point of $\hat{\mathbf{C}}$ is a limit point is the Picard group consisting of all maps

$$
z \mapsto \pm\left(\begin{array}{cc}
A & B \\
C & D
\end{array}\right), \quad A D-B C=1
$$

where $A, B, C, D$ are Gaussian integers.

The simplest Fuchsian group of the first kind is the elliptic modular group $\Gamma$ of maps (2.1) where $A, B, C, D$ are rational integers. The principal congruence subgroup modulo 2 ( of $\Gamma$ ) is defined by the additional requirement that $A$ and $D$ be odd and $B$ and $C$ even. This group is denoted by $\Gamma(2)$ and is of the first kind (for instance, because it is of finite index in $\Gamma$ ).

The group $\Gamma(2)$ is torsion free and represents (over $U$ ) a thrice punctured sphere; the canonical map $U \rightarrow U / \Gamma(2)$ is a holomorphic universal covering.

The group $\Gamma$ represents a once punctured sphere and the canonical map $U \rightarrow$ $U / \Gamma$ is branched of order 2 over 2 points.

The following construction, going back to Poincaré, yields a Fuchsian group which represents (over $U$ ) a closed Riemann surface of genus $p>1$. Let $\Pi$ be a regular non-Euclidean $4 p$-gon with all interior angles equal to $\pi / 2 p$. There is a nearly standard way of choosing $2 p$ non-Euclidean motions $A_{1}, \ldots, A_{2 p}$ (in $U$ ) such that each $A_{j}$ identifies one edge of $\Pi$ with another. The non-Euclidean motions $A_{1}, \ldots, A_{2 p}$ satisfy the relation

$$
A_{1} \circ A_{2} \circ A_{1}^{-1} \circ A_{2}^{-1} \circ \cdots \circ A_{2 p-1}^{-1} \circ A_{2 p}^{-1}=\mathrm{id},
$$

and the polygon $\Pi$, with the identifications by $A_{1}, \ldots, A_{2 p}$ carried out, becomes a closed Riemann surface $S$ of genus $p$, with complex structure compatible with that of $U$. The group $G$ generated by $A_{1}, \ldots, A_{2 p}$ is Fuchsian of the first kind and represents $S$ over $U$.

Under these circumstances one says that $\Pi$ is a fundamental domain for $G$ (in $U$ ), which means that the set-theoretical boundary $\partial \sqcap$ of $\sqcap$ has measure 0 , no two distinct points of $\operatorname{Int}(\Pi)$ are $G$-equivalent, and every point of $U$ is $G$ equivalent to some point of $\mathrm{Cl}(\Pi)$. Since $\Pi$ is also a non-Euclidean polygon we call it a fundamental polygon.

Extending the method used above one can construct a Fuchsian group of the first kind $G$ which represents (over $U$ ) a closed Riemann surface $S$ of some genus $p \geq 0$ from which one has removed $n \geq 0$ points, and such that the canonical map 
$U \rightarrow U / G=S$ is branched over $k \geq 0$ points $\zeta_{1}, \ldots, \zeta_{k}$ of $S$, of orders $\nu_{1}, \ldots$, $\nu_{k}$, respectively. The only condition to be satisfied is the inequality

$$
2 p-2+n+\left(1-\frac{1}{\nu_{1}}\right)+\cdots+\left(1-\frac{1}{\nu_{k}}\right)>0 .
$$

The sequence

$$
\left(p, n, k ; \nu_{1}, \ldots, \nu_{k}\right)
$$

is called the signature of $G$, and the pair $(p, n)$ is called the type of $G$. (This terminology is not used, or is modified, by some authors.) Every finitely generated Fuchsian group of the first kind has a signature.

Non-Fuchsian Kleinian groups can be obtained by constructing fundamental polyhedra in non-Euclidean 3-space; this also goes back to Poincaré, cf. Maskit's book for a modern presentation.

\section{Combination theorems; Schottky groups}

Let $G_{1}$ and $G_{2}$ be finitely generated Kleinian groups. Klein's combination theorem gives a sufficient condition for the group $G$ generated by $G_{1}$ and $G_{2}$ to be Kleinian and the free product of $G_{1}$ and $G_{2}$. The condition reads: $G_{1}$ and $G_{2}$ have fundamental domains $\Pi_{1}$ and $\Pi_{2}$, each bounded by one or several Jordan polygons, such that the boundary of $\Pi_{1}$ (of $\Pi_{2}$ ) is contained in $\Pi_{2}$ (in $\Pi_{1}$ ). Under this hypothesis the intersection $\Pi=\Pi_{1} \cap \Pi_{2}$ is a fundamental domain for $G$.

One owes to Maskit a far-reaching extension of this result. Maskit's first combination theorem deals with two Kleinian groups, $G_{1}$ and $G_{2}$, which have a common subgroup $G_{0}$, and gives sufficient geometric conditions for $\left\langle G_{1}, G_{2}\right\rangle$ to be the free product of $G_{1}$ and $G_{2}$, amalgamated over $G_{0}$, and Kleinian.

Maskit's second combination theorem deals with a Kleinian group $G$ and two subgroups $H_{1}$ and $H_{2}$ of $G$, which are not conjugate in $G$ but such that there is a $g \in \mathbf{M} \backslash G$ with $H_{2}=g H_{1} g^{-1}$. The theorem gives sufficient geometric conditions for $\left\langle G, g_{1}, g_{2}\right\rangle$ to be the HNNextension of $G$ by $g$, and Kleinian.

The theory of Kleinian groups may be said to have begun with Schottky's work on groups which bear his name.

Let $C_{1}, C_{1}^{\prime}, C_{2}, \ldots, C_{p}^{\prime}$ be $2 p$ disjoint Jordan curves in $\hat{\mathbf{C}}$ and assume that $C_{1}+\cdots+C_{p}^{\prime}$ is the boundary of a domain of connectivity $2 p$. Assume also that there are $p$ Möbius transformations $A_{1}, \ldots, A_{p}$ such that $A_{j}$ maps the domain exterior to $C_{j}$ onto the domain interior to $C_{j}^{\prime}$. Note that $A_{j}$ certainly exists if $C_{j}$ and $C_{j}^{\prime}$ are circles.

The group $G$ generated by $A_{1}, \ldots, A_{p}$ is free and Kleinian and the domain exterior to all circles $C_{1}, \ldots, C_{p}^{\prime}$ is a fundamental domain for $G$. The proof is easy if $p=1$ and proceeds by induction on $p$ for $p>1$; the induction argument uses Klein's combination theorem. 
A group $G$ obtained in this way is called a Schottky group of genus $p$. It is called a geometric Schottky group if it can be constructed choosing as curves $C_{1}, \ldots, C_{p}^{\prime}$ only circles. (In the older literature one says "Schottky group" and "group of Schottky type" instead of "geometric Schottky group" and "Schottky group".)

The existence of non-geometric Schottky groups was proved by Marden.

It is known that the limit set of a Schottky group has area 0. But it has positive logarithmic capacity (Myrberg). The latter property extends to all Kleinian groups since every such group must have Schottky subgroups.

Maskit proved that a (finitely generated) Kleinian group is Schottky if and only if it is free and has no parabolic elements.

The young Schottky thought that every closed Riemann surface of genus $p$ can be represented by a Schottky group of this genus. He did not publish or pursue this guess, following the advice of Weierstrass. The so-called retrosection theorem which asserts that he was right (as long as one does not restrict oneself to geometric Schottky groups) was established much later by Koebe and by Courant; we shall return to this in Section 6 .

\section{The finiteness theorem; area inequalities}

From now on we assume that $G$, with or without subscripts, denotes a nonelementary finitely generated Kleinian group. A central result in the theory is the Ahlfors finiteness theorem which asserts that $\Omega(G)$ has at most finitely many nonconjugate components $\Delta_{1}, \ldots, \Delta_{r}$, that each Riemann surface $S_{i}=\Delta_{i} / \operatorname{Stab}_{G} \Delta_{i}$ is a closed (compact) surface with at most finitely many points removed, and that the natural map $\Delta_{i} \rightarrow S_{i}$ is branched at at most finitely many points.

There are today several proofs of the finiteness theorem; they differ not in the conceptual framework but in the "hard analysis" tool used to overcome a technical difficulty. In Ahlfors' original proof this tool is a delicate "mollifier", in Sullivan's proof the tool is the theory of prime ends, and in the author's proof the tool is a lemma about quasiconformal mappings.

The author's area inequalities are quantitative refinements of the finiteness theorem. Recall that for every component $S_{i}$ of $\Omega(G) / G$ there is a branched covering map $\Delta_{i} \rightarrow S_{i}$. This map together with the Poincaré metric on $\Delta_{i}$ induces a metric, called again Poincaré metric, on $S_{i}$, and in this metric $S_{i}$ has an area $A_{i}$. The following statement is classical: if $S_{i}$ is obtained from a closed surface of genus $p_{i}$ by removing $n_{i}$ points, and if the map $\Delta_{i} \rightarrow S_{i}$ is branched over $m_{i}$ points, of orders $\nu_{i 1}, \ldots, \nu_{i m_{i}}$, respectively, then

$$
A_{i}=2 \pi\left(2 p_{i}-2+n_{i}+m_{i}-b_{i}\right)
$$

where $b_{i}$ is the sum of the reciprocal branch numbers,

$$
b_{i}=\left(1 / \nu_{i 1}\right)+\cdots+\left(1 / \nu_{i m_{i}}\right) .
$$


The area of $\Omega(G) / G$ is the sum $A_{1}+\cdots+A_{r}$.

The first area inequality asserts that if $G$ is generated by $N$ elements, then

$$
\text { Area }(\Omega(G) / G) \leq 4 \pi(N-1) \text {. }
$$

This is sharp; equality is achieved for Schottky groups.

A sharp estimate of $r$, the number of components of $\Omega(G) / G$, in terms of $N$, is not known.

Since no $A_{i}$ can be less than $\pi / 21$ (cf. (4.1) and (2.2)), the area inequality yields the trivial estimate $r \leq 84(N-1)$. Ahlfors improved this to $r \leq 18(N-1)$.

Assume now that $\Delta_{1}$ is an invariant component of $\Omega(G)$, so that $g\left(\Delta_{1}\right)=\Delta_{1}$ for all $g \in G$. The second area inequality asserts that in this case

$$
\text { Area }(\Omega(G) / G) \leq 2 \operatorname{Area}\left(\Delta_{1} / G\right) \text {. }
$$

Corollary: If $G$ has two invariant components, $\Delta_{1}$ and $\Delta_{2}$, then

$$
\Omega(G)=\Delta_{1} \cup \Delta_{2} .
$$

In this case both $\Delta_{1}$ and $\Delta_{2}$ are simply connected. We will give a more precise description of groups with two invariant components later, see Section 8.

\section{The measure of the limit set}

In his paper on the finiteness theorem Ahlfors asked whether the area $(=$ 2 -dimensional Lebesgue measure) of the limit set $\Lambda(G)$ of a finitely generated Kleinian group is zero. Later he proved that this is so if $G$, considered as a group of 3-dimensional non-Euclidean motions, has a fundamental (finitely many sided) non-Euclidean polyhedron.

Kleinian groups which have a fundamental polyhedron are now called geometrically finite; the difference between geometrically finite and geometrically infinite groups turns out to be very important.

A Kleinian group $G$ is called a function group if it has an invariant component, i.e., if $\Omega(G)$ has a component $\Delta$ such that $g(\Delta)=\Delta$ for all $g \in G$. Maskit gave a topological description of all geometrically finite function groups.

Of course, geometric finiteness is not a necessary condition for a group $G$ to have a limit set of vanishing area. There are several known sufficient conditions, for instance, Bonahan proved that mes $\Lambda(G)=0$ if $G$ is not a free product. Yet we do not know whether there is a finitely generated $G$ with mes $\Lambda(G)>0$.

Occasionally the question can be bypassed using a result of Sullivan which we proceed to describe.

A bounded measurable function $\mu(z)$ defined on $\mathbf{C}$ is called compatible with respect to a Kleinian group $G$ if

$$
\mu(g(z)) \overline{g^{\prime}(z)} / g^{\prime}(z)=\mu(z) \quad \text { a.e. } \quad \text { for } g \in G .
$$


Sullivan proved that (5.1) implies that

$$
\mu(z)=0 \quad \text { a.e. } \quad \text { on } \Lambda(G) .
$$

Of course, this result is not vacuous only for a group $G$ with mes $\Lambda(G)>0$.

(We remark, though this is of no importance for the present paper, that Sullivan's result remains valid for non-Kleinian groups, i.e., for the case when $G$ is a discrete group of Möbius transformations with $\Lambda(G)=\hat{\mathbf{C}}$.)

\section{Beltrami equations}

Let $D$ be a domain in $\mathbf{C}$. An element $\mu$ of the Banach space $L_{\infty}(D)$ is (or, more precisely, is defined by) a bounded measurable complex valued function $\mu(z), z \in D$; the norm

$$
\|\mu\|_{\infty}=\|\mu\|_{L_{\infty}(D)}
$$

is the smallest number $k$ such that $|\mu(z)| \leq k$ a.e. in $D$. If $\|\mu\|_{\infty}<1, \mu$ is called a Beltrami coefficient in $D$.

A Beltrami coefficient in $D$ defines a (not necessarily smooth) Riemannian metric on $D$,

$$
d s^{2}=\sigma^{2}|d z+\mu d \bar{z}|^{2}
$$

where $\sigma$ is a positive function: every Riemannian metric can be so represented.

A $\mu$-conformal map $w$ of $D$ onto a subdomain $D^{\prime} \subset \hat{\mathbf{C}}$ is a homeomorphic bijection $w: D \rightarrow D^{\prime}$ such that the distributional derivatives

$$
w_{z}=\frac{w_{x}-i w_{y}}{2}, \quad w_{\bar{z}}=\frac{w_{x}+i w_{y}}{2}
$$

are locally square-integrable measurable functions satisfying a.e. the Beltrami equation

$$
w_{\bar{z}}=\mu w_{z}
$$

The geometric meaning of this equation is that $w$ is a conformal map with respect to the Riemannian metric (6.1).

The existence of local homeomorphic solutions of (6.3), and the main properties of these solutions, for the case when $\mu$ is merely bounded measurable, rather than Hölder continuous, have been proved by Morrey in 1938. (Later Bojarski showed that $w_{z}$ and $w_{\bar{z}}$ are locally $L_{p}$, for some $p>2$ depending on $\|\mu\|_{\infty}$.)

Morrey's result, together with Koebe's general uniformization principle, imply that for $D=\mathbf{C}$ and for any Beltrami coefficient $\mu$ in $\mathbf{C}$ there exist a unique $\mu$ conformal automorphism $z \rightarrow w^{\mu}(z)$ of $\mathbf{C}$ onto itself which fixes the points 0,1 $($ and $\infty)$. 
A direct proof of this generalized Riemann mapping theorem, as well as the main local properties of quasiconformal maps, are contained in the 1960 paper by Ahlfors and Bers, together with the technically important additional result that, for every $z \in \mathbf{C}$, the number of $w^{\mu}(z)$ depends holomorphically on $\mu \in L_{\infty}(\mathbf{C})$.

An analogue, and a corollary of the generalized Riemann mapping theorem is the statement $\Phi$ : if $\mu$ is a Beltrami coefficient defined in the upper half-plane $U$, then there exists a unique $\mu$-conformal homeomorphism $z \rightarrow w_{\mu}(z)$ of $\hat{U}=$ $U \cup \mathbf{R} \cup\{\infty\}$ onto itself, which fixes the real axis $\mathbf{R}$, and the points $0,1, \infty$. For every fixed $z=x+i y, y \geq 0, w_{\mu}(w)$ depends real analytically on $\mu$.

A Beltrami differential $m$ on Riemann surface $S$ is a rule which assigns to every local parameter $\zeta$, defined on a plane domain $D \subset S$, a Beltrami coefficient $\mu(\zeta)$ such that the $(-1,1)$ differential $\mu d \bar{\zeta} / d \zeta$ is invariant under parameter changes. It is also required that the absolute value $|m|=|\mu|$, which is a globally defined scalar measurable function on $S$, that is, an element of $L_{\infty}(S)$, satisfy a.e. the inequality $\|\mu\|_{L_{\infty}}<1$. In these circumstances we say that $m=\mu d \bar{\zeta} / d \zeta$, locally.

Let $F$ be a bijective homeomorphism of a Riemann surface $S$ onto a Riemann surface $S_{1}$. We say that a Beltrami differential $m$ defined on $S$ is the Beltrami differential of $F$ if, for every point $P$ on $S$, for every local parameter $\zeta$ defined near and vanishing at $P$, and for every local parameter $\omega$ defined near and vanishing at $F(P)$, we have that near 0 the map $\omega \circ F \circ \zeta^{-1}$ has a Beltrami coefficient $\mu(\zeta)$, and $m=\mu(\zeta) d \bar{\zeta} / d \zeta$ locally, near 0 .

A map which has a Beltrami differential is called quasiconformal.

\section{Quasiconformal deformations of Kleinian groups}

Let $G$ be as before a Kleinian group and $\mu$ a Beltrami coefficient on $\hat{\mathbf{C}}$ compatible with $G$, i.e., satisfying relation (5.1) for every $g \in G$. Sullivan's theorem stated in Section 5 implies that it suffices to require that (5.1) hold a.e. in $\Omega(G)$.

A direct calculation shows that, for every $g$ in $G$, the mapping $\omega^{\mu} \circ g$ satisfies (a.e.) the same Beltrami equation as $w^{\mu}$. But two $\mu$-conformal maps differ only by a conformal one, so that

$$
w^{\mu} \circ g=g^{\mu} \circ w^{\mu}
$$

where $g^{\mu}$ is a holomorphic function. But

$$
g^{\mu}=w^{\mu} \circ g \circ\left(w^{\mu}\right)^{-1}
$$

is clearly a topological self-map of $\hat{\mathbf{C}}$ and thus an element of $\mathbf{M}$.

One sees at once that the group

$$
G^{\mu}=w^{\mu} G\left(w^{\mu}\right)^{-1}
$$


is a Kleinian group, that $w^{\mu}(\Omega(G))=\Omega\left(G^{\mu}\right)$ and $w^{\mu}(\Lambda(G))=\Lambda\left(G^{\mu}\right)$. The isomorphism

$$
g \mapsto w^{\mu} \circ g \circ\left(w^{\mu}\right)^{-1}=g^{\mu}
$$

is called a quasiconformal deformation of $G$ onto $G^{\mu}$, induced by $\mu$.

This construction can be reversed, and this suggests a method for proving uniformization theorems, called variation of conformal structure or, less formally, the $\mu$-trick.

As an example we prove the classical retrosection theorem: every closed Riemann surface $S$ of genus $p>1$ can be uniformized by a Schottky group, that is, there is a Schottky group $G$ with $\Omega(G) / G$ conformal to $S$.

Repeating the construction of a Schottky group in Section 3 , we choose $2 p$ disjoint compact circular discs on $\mathbf{C}$, call them $D_{01}, D_{01}^{\prime}, \ldots, D_{0 p}, D_{0 p}^{\prime}$ and $p$ Möbius transformations $A_{01}, \ldots, A_{0 p}$ such that (i) the complement in $\hat{\mathbf{C}}$ of $D_{01}+D_{01}^{\prime}+\cdots+D_{0 p}^{\prime}$ is a plane domain of connectivity $2 p$, and (ii) $A_{0 j}$ maps the interior of $D_{0 j}$ onto the exterior of $D_{0 j}^{\prime}, j=1, \ldots, p$. The group $G_{0}$ generated by $A_{01}, \ldots, A_{0 p}$ is a Schottky group. It is not difficult to construct a smooth (say of class $\left.C^{2}\right)$ quasiconformal map of $S_{0}=\Omega\left(G_{0}\right) / G_{0}$ onto the given Riemann surface $S$. This map has a Beltrami differential $\mu$ defined first over some fundamental domain for $G_{0}$ and then extended over all of $\Omega\left(G_{0}\right)$ by the condition

$$
\mu(g(z)) \overline{g^{\prime}(z)}=\mu(z) g^{\prime}(z)
$$

for $z \in \Omega\left(G_{0}\right)$ and $g \in G_{0}$. Since $\mu$ is compatible with the group $G_{0}$, the group

$$
G=w^{\mu} G_{0} \circ\left(w^{\mu}\right)^{-1}
$$

is a Kleinian group, more precisely a Schottky group, and $\Omega(G) / G$ is conformally equivalent to the given Riemann surface $S$.

We do not discuss the additional conditions which must be imposed in order to make the uniformizing Schottky group $G$ unique.

\section{Quasi-Fuchsian groups}

A quasi-Fuchsian group (of the first kind) $G$ is a Kleinian group whose limit set $\Lambda(G)$ is a Jordan curve on $\hat{\mathbf{C}}$. We shall consider only finitely generated quasiFuchsian groups $G$, and shall assume, for the sake of simplicity, that $G$ has no elliptic or parabolic elements.

It is often convenient to orient the Jordan curve $\Lambda(G)$ and to assume that it passes through the points $0,1, \infty$ (in this order). The two simply connected components of $\hat{\mathbf{C}} \backslash \Lambda(G)$ will be denoted by $\tilde{U}(G)$ and $\tilde{L}(G), \tilde{U}(G)$ being to the left of $\Lambda(G)$. A Fuchsian group is also quasi-Fuchsian, with $\Lambda(G)=\mathbf{R} \cup\{\infty\}$ and $\tilde{U}(G)=U$. A quasi-Fuchsian group $G$ represents two closed Riemann surfaces,

$$
S_{1}=\tilde{U}(G) / G \quad \text { and } \quad S_{2}=\tilde{L}(G) / G .
$$


The theorem on simultaneous uniformization (or, rather a special case of this theorem) asserts that $S_{1}$ and $S_{2}$ may be prescribed arbitrarily, except that they must have the same genus $p>1$.

The only proof I know involves the $\mu$-trick (whereas all uniformization theorems mentioned before have also "classical" proofs which do not depend on quasiconformal mappings).

Sketch of proof. Let $G_{0}$ be a Fuchsian group such that $U / G_{0}$ is (conformal to) the given surface $S_{1}$ (the existence of $G_{0}$ can be proved by the method in Section 6). Let $L$ denote the lower half-plane. Then $L / G_{0}$ is conformal to the mirror image $\bar{S}_{1}$ of $S_{1}$. (The points and open sets of $\bar{S}_{1}$ are those of $S_{1}$, the local parameters on $\bar{S}_{1}$ are the complex conjugates of the local parameters on $S_{1}$.) Considered as a quasi-Fuchsian group $G_{0}$ has the components $U$ and $L$. The quotient $U / G_{0}$ is already what we want it to be, the complex structure of $L / G_{0}$ is to be changed into that of $S_{2}$ by the $\mu$-trick.

There is a quasiconformal homeomorphism $f$ of $\bar{S}_{1}=L / G$ onto $S_{2}$. It can be lifted to a homeomorphism $\tilde{f}$ of $\bar{S}_{1}$ onto the universal covering surface of $S_{2}$. Let $m$ be the Beltrami differential of the map which coincides with the identity on $U$ and with the map $\tilde{f}$ on $L$. This $m$ is compatible with $G_{0}$. One verifies, as in Section 6, that

$$
G=w^{\mu} G_{0}\left(w^{\mu}\right)^{-1}
$$

is a quasi-Fuchsian group with $\tilde{U}(G) / G$ conformally equivalent to $S_{1}$ and $\tilde{L}(G) / G$ to $S_{2}$.

Remark 1. We can now answer a question hinted at at the end of Section 4. A finitely generated Kleinian group with two invariant components is quasi-Fuchsian.

Remark 2. Bowen proved that the Hausdorff dimension of the limit set of a quasi-Fuchsian not Fuchsian group is strictly between 1 and 2 .

\section{Teichmüller spaces}

The Teichmüller space $T(G)$ of a Fuchsian group $G$ is the space of normalized quasiconformal deformations of $G$. For the sake of simplicity we assume, as before, that $G$ is finitely generated, purely hyperbolic and of the first kind; then $S=U / G$ is a closed Riemann surface of some genus $p>1$.

We denote by $Q$ the group of all quasiconformal self-mappings of the closed upper half-plane $\hat{U}=U \cup \mathbf{R} \cup\{\infty\}$, by $Q_{n}$ the subgroup which fixes the points $0,1, \infty$, and by $Q_{0}$ the normal subgroup which fixes every $x \in \hat{\mathbf{R}}=\mathbf{R} \cup\{\infty\}$. We say that $u \in Q(G)$ if $u \in Q$ and $u G u^{-1}$ is again a Fuchsian group. Every element $u$ of $Q(G)$ induces a quasiconformal deformation

$$
G \ni g \mapsto u \circ g \circ u^{-1}=\hat{g} \in u G u^{-1}
$$


this deformation is called normalized if $u \in Q_{n}$. Two deformations induced by two elements $u_{1}$ and $u_{2}$ are identical if and only if $u_{1}$ and $u_{2}$ coincide on $\hat{\mathbf{R}}$. The deformation induced by $u$ will be denoted by $[u]$; thus $[u]$ determines and is determined by $u \mid \hat{\mathbf{R}}$. The space $T(G)$ is the space of all $[u], u \in Q_{n}(G)$.

Every $u \in Q(G)$ induces an allowable bijection of $T\left(u g u^{-1}\right)$ onto $T(G)$ which takes

$$
[w] \in T\left(u g u^{-1}\right)
$$

onto

$$
\left[\alpha \circ w \circ u^{-1}\right] \in T(G),
$$

where $\alpha$ is a real Möbius transformation chosen so that $\alpha \circ w \circ u^{-1}$ fixes 0,1 and $\infty$. The allowable self-mappings of $T(G)$ form the modular group $\operatorname{Mod}(G)$ of $G$ and

$$
X(G)=T(G) / \operatorname{Mod}(G)
$$

is the set of PSL $(2 \mathbf{R})$ conjugacy classes of quasiconformal images of $G$.

There is a canonical way of representing $T(G)$ as a bounded domain in a complex vector space, namely the space $B_{2}(L, G)$ of bounded automorphic forms of weight $(-4)$ for the group $G$, acting on the lower half-plane. The elements of this vector space are holomorphic functions $\varphi(z), z \in L$, satisfying the functional equation

$$
\varphi(g(z)) g^{\prime}(z)^{2}=\varphi(z), \quad(g \in G, z \in L) .
$$

Thus $\varphi(z) d z^{2}$ is a holomorphic quadratic differential on the Riemann surface $L / G$. The Riemann-Roch theorem implies that

$$
\operatorname{dim} B_{2}(L, G)=3 p-3 .
$$

One defines in this space the norm

$$
\|\varphi\|=\sup \left|y^{2} \varphi(x+i y)\right|
$$

where $-\infty<x<+\infty,-\infty<y<0$. (Note that $\left|y^{2} \varphi(z)\right|$ is $G$-invariant.)

We now proceed to define a mapping of $T(G)$ into $B_{2}(L, G)$. Let $\tau$ be an element of $T(G)$. There is a Beltrami coefficient $\mu$ for $G$, defined in $U$, and compatible with $G$, such that $\tau=w_{\mu} \mid \hat{\mathbf{R}}=\left[w_{\mu}\right]$. We continue $\mu$ over all of $\mathbf{C}$ by setting

$$
\mu_{0}(z)= \begin{cases}\mu(z) & \text { for } z \in U \\ 0 & \text { for } z \in L .\end{cases}
$$

The Beltrami coefficient $\mu_{0}$ is also $G$-compatible, and one can verify that for any two Beltrami coefficients $\mu$ and $\nu$, defined in $U$ and compatible with $G$ the following statements are equivalent. 
(i) $\left[w_{\mu}\right]=\left[w_{\nu}\right]$,

(ii) $w^{\mu_{0}}\left|L=w^{\nu_{0}}\right| L$

(iii) $w^{\mu_{0}}\left|\hat{\mathbf{R}}=w^{\nu_{0}}\right| \hat{\mathbf{R}}$.

Now $w^{\mu_{0}} \mid L$ is a schlicht holomorphic function in $L$, so that it is natural (at least for a complex analyst) to form the Schwarzian derivative of $w^{\mu_{0}} \mid L$ which we denote by $\varphi^{\mu}$; thus

$$
\varphi^{\mu}=\{w, z\}=\left(w^{\prime \prime} / w^{\prime}\right)^{\prime}-\frac{1}{2}\left(w^{\prime \prime} / w^{\prime}\right)^{2},
$$

where $w=w^{\mu_{0}} \mid L$.

One can verify that $\varphi^{\mu}(z)$ satisfies relation (9.1) and thus belongs to $B_{2}(L, G)$. Also, since $\varphi^{\mu}$ is the Schwarzian derivative of a schlicht function in $L$, we conclude from the Kraus-Nehari inequality that

$$
\|\varphi\| \leq \frac{3}{2}
$$

It can be shown that the map

$$
T(G) \rightarrow B_{2}(L, G)
$$

which takes $w_{\mu} \mid \mathbf{R}$ into $\varphi^{\mu}$ is a bijection of $T(G)$ onto a subdomain of the ball $\|\varphi\|<3 / 2$ in $B_{2}(L, G)$, and there is a unique natural way of defining a complex structure in $T(G)$ such that (9.3) becomes a holomorphic homeomorphism. The same is then true of every element of the modular group and of every allowable mapping.

It is usually convenient to identify $T(G)$ with its image under the mapping (9.3). The image contains the open ball $\|\varphi\|<1 / 2$ in $B_{2}(L, G)$. This statement is a corollary of the elegant Ahlfors-Weil lemma which can be restated as follows.

Let $\psi(z)$ be a holomorphic quadratic differential in $L$, for the group $G$, and assume that $\|\psi\|<1 / 2$, i.e., that $2|\psi(z)|<y^{-2}$ for $z \in L$. Set

$$
\begin{cases}\mu(z)=-2 y^{2} \psi(\bar{z}) & \text { for } z \in L \\ \mu(z)=0 & \text { for } z \in U .\end{cases}
$$

Then $\mu(z)$ is a Beltrami coefficient compatible with $G$ in $\mathbf{C}$, and the Schwarzian derivative of $w^{\mu} \mid L$ is $\psi=\varphi^{\mu}$.

We conclude this section by noting that $T(G)$ is a cell (this follows, for instance from Teichmüller's mapping theorem) and that $T(G)$ is holomorphically convex (there are, by now, several proofs of this fact).

Remark 1. Practically all results of this and the preceding section remain valid when the Fuchsian group $G$ is not finitely generated, or when it contains elliptic or parabolic elements, or when it is of the second kind. This may require some modifications of definitions (for instance, the finite dimensional space $B_{2}(L, G)$ may have to be replaced by an infinitely dimensional Banach space). Also, some proofs may become significantly harder. 
Remark 2. We did not yet mention Teichmüller spaces of Riemann surfaces. Assuming their definition known, and assuming $G$ to be torsion free and of the first kind, we can state a basic theorem.

There exists a canonical isomorphism between the Teichmüller space $T(G)$ and the Teichmüller space of the Riemann surface $U / G$.

A modified isomorphism theorem can be proved if $G$ is of the second kind and/or has torsion.

\section{Boundary groups}

Let $G, B_{2}(L, G), T(G)$ have the same meaning as in the preceding section, and let $\Psi$ denote the bijection (9.3). We identify $T(G)$ with its image under $\Psi$ and denote by $\mathrm{Cl}(T(G))$ the closure of $T(G)=\Psi(T(G))$ in $B_{2}(L, G)$; then $\partial T(G)=\mathrm{Cl}(T(G)) \backslash T(G)$ is the set theoretical boundary of $T(G)$ in $B_{2}(L, G)$. We will associate a Kleinian group to every point of $\partial(T(G))$.

If $\psi \in B_{2}(L, G)$ is given, we denote by $\eta_{j}(z), j=1,2$, the two solutions of the ordinary differential equation

$$
2 \eta^{\prime \prime}(z)+\eta(z) \psi(z)=0,
$$

subject to the initial conditions

$$
\eta_{1}=\eta_{2}^{\prime}=1, \quad \eta_{1}^{\prime}=\eta_{2}=0, \quad \text { at } \quad z=-i .
$$

These two functions are holomorphic in $L$, and depend continuously on $\psi \in$ $B_{2}(L, G)$. The quotient

$$
V^{\psi}(z)=\eta_{1}(z) / \eta_{2}(z)
$$

is a locally single-valued meromorphic solution of the Schwarzian differential equation

$$
\left\{V^{\psi}(z), z\right\}=\psi(z)
$$

Comparing this with (9.2) we conclude that there exists a Möbius transformation $\alpha_{\psi}$ (dependent on $\psi$ ) such that

$$
w^{\mu_{0}}=\alpha_{\psi} \circ V^{\psi}
$$

This is valid for $\psi \in T(G)$ or, more precisely, for $\Psi(\psi) \in T(G)$. Indeed $w^{\mu_{0}}$ was defined assuming that $\left[w_{\mu}\right] \in T(G)$. Also, if $\psi$ converges to a point $\hat{\psi}$ on $\partial T(G)$, there is no reason to assume that $\alpha_{\psi}$ converges.

On the other hand, $V^{\psi}$ is defined for all $\psi$ in $B_{2}(G)$ and it makes sense to study the group

$$
G^{\psi}=V^{\psi} G\left(V^{\psi}\right)^{-1}, \quad \psi \in \partial T(G) .
$$


Such groups are called boundary groups (of $G$ ). The facts are as follows.

The group $G^{\psi}$ is Kleinian and has a single simply connected invariant component. (Groups with the latter property are called $b$-groups. It is not known whether every $b$-group is a boundary group.)

In general (in the sense of measure or in the sense of dimension) $G^{\psi}$ has no other components. Such groups are called (totally) degenerated. Totally degenerated groups are geometrically infinite (cf. Section 5). As a matter of fact, this is how geometrically infinite groups were discovered.

A function group which is not totally degenerated has non-invariant components. Assume that $D_{1}, \ldots, D_{n}$ is a complete list of non-conjugate non-invariant components, and let $\Gamma_{j}$ be the stabilizer of $D_{j}$. Also set $S_{j}=D_{j} / \Gamma_{j}$. Then each $S_{j}$ has at least one puncture, and if the non-Euclidean area of $S_{j}$ is $A_{j}$, we have that

$$
A_{1}+\cdots+A_{r} \leq A
$$

where $A$ is the area of $L / G$. (This is simply the second area inequality, cf. Section 4.) The group $G^{\psi}$ is called partially degenerate (or regular) according to whether (10.1) is valid with the strict inequality sign (or with the equality sign).

The terms "regular", "partially degenerated" and "totally degenerated" make sense for any $b$-group. Abikoff proved that regular $b$-groups are boundary groups.

\section{Selected references}

AвікоғF, W.: The real analytic theory of Teichmüller spaces. - Lecture Notes in Mathematics 820. Springer-Verlag, Berlin-Heidelberg-New York, 1980.

Ahlfors, L.V.: Lectures on quasiconformal mappings. - Van Nostrand, Princeton, 1966.

AHLFors, L.V.: Quasiconformal mappings, Teichmüller spaces and Kleinian groups. Proceedings of the International Congress of Mathematicians, Helsinki, 1978, Vol. 1, 71-84.

BERs, L.: Uniformization, moduli and Kleinian groups. - Bull. London Math. Soc. 4, 1972, 257-300.

BERs, L.: Finite dimensional Teichmüller spaces and generalizations. - Bull. Amer. Math. Soc. 5, 1981, 131-172.

BERS, L., and I. KRA: A crash course on Kleinian groups. - Lecture Notes in Mathematics 400. Springer-Verlag, Berlin-Heidelberg-New York, 1974.

Fathi, A., F. Laudenbach, and V. Poenaru: Travaux de Thurston sur les surfaces. Astérisque 66-67, 1979.

Gardiner, F.P.: Teichmüller theory and quadratic differentials. - John Wiley and Sons, New York-Chichester-Brisbane-Toronto-Singapore, 1987.

HARVEY, W.J.: Discrete groups and automorphic functions. - Academic Press, LondonNew York-San Francisco, 1977.

KrA, I.: Automorphic forms and Kleinian groups. - Benjamin, Reading, Mass., 1972.

LEHTo, O.: Univalent functions and Teichmüller spaces. - Graduate Texts in Mathematics 109. Springer-Verlag, Berlin-Heidelberg-New York, 1987. 
Lehto, O., and K. Virtanen: Quasiconformal mappings in the plane, 2nd edition. Springer-Verlag, Berlin-Heidelberg-New York, 1973.

MASKIT, B.: Kleinian groups. - Springer-Verlag, Berlin-Heidelberg-New York-LondonParis-Tokyo, 1988.

NAG, S.: The complex-analytic theory of Teichmüller spaces. - John Wiley and Sons, New York-Chichester-Brisbane-Toronto-Singapore, 1988.

ThuRston, W.P.: On the geometry and dynamics of diffeomorphisms of surfaces. - Bull. Amer. Math. Soc. 19, 1988, 417-431.

Columbia University

Department of Mathematics

New York, NY 10027

U.S.A.

and

City University of New York

Graduate Center

New York, NY 10036

U.S.A.

Received 6 February 1989 